\title{
Theoretisieren in der Soziologie Bezugskontexte und Modi soziologischer Theoriebildung
}

\author{
Sebastian Büttner (FAU Erlangen-Nürnberg) ${ }^{1}$
}

\begin{abstract}
Die Grundlagen des Theoretisierens werden in der Soziologie bisher kaum reflektiert. Unterschiedliche Vorstellungen zum Wesen von Theorie(n) erschweren zudem eine systematische Auseinandersetzung mit der Praxis der Theoriebildung. Der Beitrag adressiert dieses Desiderat, indem wir die Grundprobleme des Theoretisierens in der Soziologie und zentrale Modi soziologischer Theoriebildung herausarbeiten. Wir grenzen uns hierbei von Vorstellungen empirischer Theoriebildung und insbesondere von Richard Swedbergs theorizingKonzeption ab, da diese die Vielfalt von Theoriearbeit in der Soziologie unterschätzen. Die Praxis des Theoretisierens lässt sich nicht auf eine einzige Art und Weise der Theoriebildung reduzieren, denn es greifen stets verschiedene Ebenen, Elemente und Bezugsprobleme theoretischer Reflexion ineinander. Auf dieser Basis unterscheiden wir abschließend sechs Modi des Theoretisierens, die die Theoriearbeit in der Soziologie grundlegend prägen und die sich nicht aufeinander reduzieren lassen.
\end{abstract}

SCHLAGWÖRTER: Theoriebildung; Soziologische Theorie; Theoriebegriff; Theoriearbeit; Theorizing; Theorienstreit

\section{Einleitung}

Die Soziologie pflegt ein ambivalentes Verhältnis zur eigenen Theorie. Einerseits haben Theoriediskussionen und theoretische Generalisierungen in der Soziologie traditionell einen hohen Stellenwert. Die Qualität soziologischer Publikation wird häufig danach bewertet, ob die ,theoretische Einbettung' gelingt oder ob ein ,origineller' Theoriebeitrag geleistet wird. Blickt man jedoch auf die Vielfalt soziologischer Theorien und unterhält man sich mit Kolleg*innen über deren Verständnis von Theorie, stellt man andererseits schnell fest, dass es sehr unterschiedliche und häufig auch sehr diffuse Vorstellungen über das Theoretisieren gibt. Mehr noch: Es herrscht weitgehend Unklarheit darüber, welchen Stellenwert Theorie in der

\footnotetext{
${ }^{1}$ Der Beitrag basiert auf gemeinsamen Diskussionen mit André Armbruster (Universität Duisburg-Essen) und auch einigen Vorarbeiten, die zusammen geleistet wurden. Ich danke, André Armbruster für seinen Input und für seine Unterstützung.
} 
soziologischen Forschungspraxis hat und was Theoriearbeit in der Soziologie letztlich bedeutet. Zudem gibt es diffuse Vorstellungen darüber, nach welchen Standards und Kriterien Theorien in der Soziologie zu entwickeln sind: Geht es lediglich um das Aufstellen und Testen von Hypothesen oder gibt es eine eigenständige, von Hypothesentests unabhängige Theoriearbeit? Lassen sich Theorien weiterentwickeln, und wenn ja, wie? Und selbst die Frage, wie Theorien eigentlich entstehen, wird erst seit kurzem gestellt (siehe für einen zögerlichen Start der Debatte: Swedberg 2014a, 2014b; Farzin/Laux 2016; Anicker 2020). Auch die Theorieausbildung beschränkt sich - wenn überhaupt - hauptsächlich auf die Vermittlung der Theoriegeschichte und der zentralen Begriffe und Theoreme bekannter Theoretiker und noch recht weniger Theoretikerinnen. Eine systematische Auseinandersetzung damit, wie man eine Theorie erstellt, wie man im wahrsten Sinne des Wortes theoretisiert, findet in der Soziologie kaum statt. Es gibt somit ein großes Missverhältnis zwischen der Erwartung an theoretischer Fundierung soziologischer Forschung und der tatsächlichen Auseinandersetzung mit den Grundlagen des Theoretisierens. Daraus folgt, dass das Theoretisieren bis heute vor allem als individuelle Leistung, als besondere Fähigkeit, wenn nicht gar als exklusive Begabung und damit als ein weitgehend „implizites Wissen“ (Polanyi 1985) angesehen wird, nicht aber als systematisch vermittel- und erlernbar. ${ }^{2}$

Ziel dieses Aufsatzes ist es, die Grundlagen soziologischer Theoriebildung und die verschiedenen Modi des Theoretisierens in der Soziologie explizit in den Blick zu nehmen, um erste Schritte in Richtung einer soziologischen Selbstaufklärung über Theoriearbeit und die Praxis des Theoretisierens zu beschreiten. Wir gehen zunächst auf die zentralen Konfliktlinien soziologischer Theoriedebatten und auf die Vielfalt soziologischer Theoriebegriffe ein, die die Vorstellungen von Theoriearbeit in der Soziologie maßgeblich prägen (Kapitel 2). Wir beleuchten anschließend Ansätze empirischer Theoriebildung, insbesondere Richard Swedbergs theorizingKonzeption, die in den vergangenen Jahren eine lebhafte internationale Debatte über Theoriebildung in der Soziologie angestoßen hat (Kapitel 3). In Abgrenzung zu Swedberg und den Ansätzen empirischer Theoriebildung erarbeiten wir im vierten Kapitel eine Systematik, die der Komplexität der Theoriearbeit in der Soziologie Rechnung trägt. Denn die Theoriearbeit erschöpft sich längst nicht nur auf eine einzige Form empirisch fundierter Theoriebildung. Die

\footnotetext{
${ }^{2}$ Ganz im Sinne Polanyis (1985: 16) und der dargestellten Ambivalenz der Theorie in der Soziologie gibt es demnach eine Diskrepanz zwischen dem Wissen um Theorie (knowing that) und dem ,Können von Theorie' im Sinne eines Wissens, wie man mit Theorie(n) arbeitet und wie man letztlich theoretisiert (knowing how).
} 
Praxis des Theoretisierens umfasst so viel mehr. Und dieses Mehr, so argumentieren wir im vierten Kapitel, ergibt sich aus der Vielfalt theoretischer Reflexion im Nexus von Theorie und Empirie sowie aus der Verschiedenheit der Geltungsansprüche und Bezugspunkte soziologischer Theoriebildung, die letztlich auch unterschiedliche Modi des Theoretisierens implizieren. Auf dieser Basis differenzieren wir schließlich sechs Modi soziologischer Theoriebildung, die die Theoretisierungspraxis in der Soziologie unseres Erachtens ganz wesentlich charakterisieren und die sich nicht gegeneinander ausspielen lassen. Im abschließenden Fazit geben wir einen Ausblick über mögliche weitere Anschlüsse an die hier vorgestellten Grundüberlegungen zum Theoretisieren in der Soziologie.

\section{Die Vielfalt von Theoriebegriffen in der Soziologie: Ein Erkenntnishindernis für ein tieferes}

\section{Verständnis soziologischer Theoriebildung?}

Vordergründig betrachtet scheint es in der Soziologie eine große Einigkeit bei der Einschätzung zentraler Theorieentwicklungen zu geben. Zumindest lässt sich beobachten, dass allein im deutschsprachigen Raum in den vergangenen Jahrzehnten eine ganze Reihe von Lehrbüchern und Sammelbänden erschienen ist, die einen Überblick über die soziologische Theorieentwicklung von ,den Klassikern' bis hin zu ,neueren Theorieentwicklungen' vermitteln (vgl. Morel et al. 1989; Treibel 1997; Haller 1999; Kaesler 2003, 2005, 2007; Münch 2004a, 2004b, 2004c; Joas/Knöbl 2004; Kneer/Schroer 2009; Rosa et al. 2009; Schroer 2017). ${ }^{3}$ So unterschiedlich diese Lehrbücher und Sammelbände im Einzelnen aufgebaut und konzipiert sind, so sehr gibt es doch eine weitgehende Übereinstimmung bei der Auswahl und Darstellung der wichtigsten Paradigmen. Bei genauerer Betrachtung muss man jedoch feststellen, dass dieser Eindruck trügt. In der Soziologie herrscht bis heute eine große Uneinigkeit im Hinblick auf grundlegende theoretische und wissenschaftstheoretische Fragen. Es gibt weder einen einheitlichen und von allen geteilten Theoriebegriff, noch gibt es eine Übereinstimmung bei der Einschätzung der Rolle der Theorie in der soziologischen Praxis (vgl. Joas/Knöbl 2004: 13ff.; Abbott 2007; Abend 2008; Albert/Sigmund 2011; Martin 2015). Ferner sind die zum Teil heftigen Grundsatzdebatten wohl auch dafür verantwortlich, dass Prozesse des Theoretisierens in der Soziologie kaum thematisiert wird. Die Diskussionen über das adäquate Verständnis von Theorie

\footnotetext{
${ }^{3}$ Diese Auswahl erhebt keinen Anspruch auf Vollständigkeit. Für den englischsprachigen Raum lässt sich jedoch eine ähnliche Entwicklung beobachten (vgl. Alexander 1987; Abrahamson 1990; Turner 1986, 2001, 2012; Giddens/Turner 1988; Dillon 2003; Benzecry et al. 2017).
} 
scheinen ein grundlegendes Nachdenken über Theoriearbeit in der Soziologie zu verhindern. Sie sind in diesem Sinne obstacles épistémologiques (Bachelard 2016) der soziologischen Theoriedebatte.

Die verschiedenen Kontroversen verdichten sich im Wesentlichen auf drei zentrale Konfliktlinien: Eine starke Trennlinie verläuft erstens zwischen Verfechter*innen einer überwiegend empirisch ausgerichteten Soziologie und jenen, die vor allem die Arbeit mit und an Theorien in den Mittelpunkt stellen. In ihrer jeweils stärksten Ausprägung bilden beide Lager im wörtlichen Sinne Parallelwelten, die keinerlei Prämissen teilen und in denen völlig unterschiedliche Vorstellungen und Standards von soziologischer Forschung, von Theoriebildung sowie von wissenschaftlicher Arbeit insgesamt kultiviert werden. Die Kontroversen reichen jedoch auch über das Verhältnis von Theorie und Empirie hinaus. Denn eine zweite Konfliktlinie verläuft entlang der Frage, was eine gute soziologische Theorie auszeichnen sollte und was nicht. Dies war und ist eine Diskussion, die auch stark mit der Debatte über die Notwendigkeit der Integration und Vereinheitlichung unterschiedlicher Paradigmen verknüpft ist (vgl. Parsons 1951, 1968; Luhmann 1984; Giddens 1988; Coleman 1990; Habermas 1995; Esser 2011).

Zudem hat sich drittens gegenwärtig eine zentrale Konfliktlinie zwischen Verfechter*innen einer im weitesten Sinne „kulturtheoretisch“ oder „kulturalistisch“ orientierten Soziologie (Reckwitz 2006; Kalthoff et al. 2008; Schmidt 2012; Knoblauch 2017) und einer hauptsächlich szientistisch und möglichst nah an naturwissenschaftlichen Standards und Methoden ausgerichteten „analytischen“ und „erklärenden“ Soziologie etabliert (Diekmann/Voß 2004; Braun 2008; Hedström 2008; Elster 2010; Esser 2011; Kroneberg 2011). Während sich die Vertreter*innen des kultursoziologischen Paradigmas im Wesentlichen auf einen sehr breiten Theoriebegriff berufen, der sich an klassischen Sozialtheorien sowie an allgemeineren philosophischen und transdisziplinären Debatten in den Kulturwissenschaften orientiert, ${ }^{4}$ propagiert die erklärende Soziologie ein ganz bestimmtes Konzept von Theoriebildung: Im Zentrum steht hier die Modellierung und nach Möglichkeit auch die Vorhersage menschlicher Entscheidungssituationen. Eine Theorie, die keine Ansätze für Erklärungen nach dem MikroMakro-Modell (Coleman 1990) und dem deduktiv-nomologischen Schema im Sinne Hempel und Oppenheims (1948) liefert, wird aus dieser Perspektive nicht als Theorie anerkannt (vgl. Esser

\footnotetext{
${ }^{4}$ Die Beiträge in Kalthoff et al. (2008) postulieren sogar eine starke Wahlverwandtschaft von allgemeiner soziologischer Theoriebildung und kulturtheoretisch fundierter qualitativer Forschung. Dies wird in ihrem Sammelband unter dem Stichwort „Empirische Theorie“ verhandelt.
} 
1993: 57ff.; Braun 2008). Zudem stützt man sich in Anlehnung an wissenschaftstheoretische Grundlagen des Kritischen Rationalismus Karl Poppers (2005) auf die Grundannahme, dass Theorien prinzipiell einer kritischen Überprüfung zu unterziehen sind und, wenn nötig, als falsch verworfen werden sollten.

Es ist nicht unser Anliegen, die Verästelungen der einzelnen Debatten weiter zu explizieren und dabei entsprechende Positionskämpfe zu replizieren. Es sollte lediglich verdeutlicht werden, dass es in der Soziologie sehr unterschiedliche Vorstellungen über Theorie und damit verbunden auch über, gute' und ,adäquate' Theoriearbeit gibt. Mit jeder der genannten Positionen verbinden sich unterschiedliche methodologische und wissenschaftstheoretische Vorentscheidungen. Daher gehören Kämpfe und Auseinandersetzungen um das, richtige' Wissenschaftsverständnis und um die adäquate Theorie in besonderer Weise zur Soziologie. Dies hat zum einen - dies zeigte bereits die klassische Wissenschaftssoziologie - mit der starken sozialen Organisation von Paradigmen und Theorietraditionen zu tun, die sich nicht so leicht von der Kritik alternativer Schulen und Deutungsangebote erschüttern lassen (siehe grundlegend dazu: Kuhn 1976). Die besondere Prävalenz theoretischer Richtungsstreits in der Soziologie ergibt sich des Weiteren aus der schieren Vielfalt soziologischer Gegenstände sowie aus der eigentümlichen Zwischenstellung der Soziologie zwischen den geistes- und naturwissenschaftlichen Denk- und Herangehensweisen (Abbott 2007). ${ }^{5}$

Die Vielfalt soziologischer Perspektiven und Methodologien führt letztlich auch zu einer Vielfalt sehr unterschiedlicher Theorieverständnisse. Dies haben Gabriel Abend (2008) und John Levi Martin (2015) in ihren Analysen vorherrschender Theoriebegriffe in der Soziologie anschaulich gezeigt. Beide arbeiten unterschiedliche Bedeutungen des Theoriebegriffs heraus, die je nach Kontext und wissenschaftstheoretischer Grundposition stark voneinander abweichen. Abend (2008: 177ff.) unterscheidet in einer - wie er es nennt - „semantischen Analyse“ soziologischer Theoriebegriffe insgesamt sieben verschiedene Bedeutungsdimensionen. Sie reichen von einem Verständnis von Theorie als ein „,[...] logically-connected system of general propositions“ (Abend 2008: 177), das Variablen miteinander in Beziehung setzt (theory $y_{1}$, über Theorie als eine hermeneutische Praxis zur Interpretation der sozialen Welt (theory $y_{3}$ ) bis hin zu zur Diskussion maßgeblicher Annahmen und Prinzipien (theory7) (für einen Überblick über alle sieben Begriffe siehe Abbildung 1). Martin (2015: 1ff.) unterscheidet die vorherrschenden Theorietypen weniger nach der Semantik, sondern nach typischen Verwendungsweisen von Theorien. Er kommt

\footnotetext{
${ }^{5}$ Ausführlicher dazu auch in einer früheren Ausgabe der ZTS: Adloff/Büttner 2013.
} 
insgesamt auf sechs Theoriebegriffe, die sich in vielen Punkten durchaus mit Abends Typologie überschneiden. ${ }^{6}$ Daneben gibt es aber auch Unterscheide. So betont Martin etwa, dass die überwiegend exegetische Rekonstruktion und Kommentierung von Theorien, die "TheoryOlogy" (Martin 2015: 2), in ihrer Bedeutung seit den 1980er Jahren enorm zurückgegangen ist und in der Soziologie immer weniger als eigenständige Forschungspraxis wertgeschätzt wird. Stattdessen macht Martin angesichts der Tendenz zur Negierung oder Geringschätzung theoretischer Grundlagenarbeit vor allem eine Zunahme der empirischen Übergeneralisierung aus, bei der klassische Standards solider Begriffs- und Theoriearbeit über Bord geworfen werden. $^{7}$

\section{Abbildung 1 ungefähr hier}

Welche Folgen hat die Vielfalt an Theoriebegriffen nun aber für das Verständnis von Theoriearbeit und insgesamt für den Umgang mit Theorie(n) in der Soziologie? Gibt es aufgrund der Vielfalt unterschiedlicher Perspektiven und Paradigmen tatsächlich keinerlei Maßstäbe und Richtlinien für Theoriearbeit? Oder lassen sich doch Kriterien für ,gute' oder zumindest ,fruchtbare' Theoriearbeit benennen? Ein Blick auf die einschlägige Literatur zeigt, dass es durchaus eine ganze Reihe von Richtlinien und Standards gibt. Sie sind jedoch in der Regel nicht das Ergebnis soziologischer Theoriedebatten und sie sind kaum (oder zumindest nicht explizit) Gegenstand der Theorieausbildung. ${ }^{8}$ Denn die Vermittlung von Grundlagen der Theoriebildung findet in der Soziologie, wie wir nachfolgend weiter ausführen, vor allem in der Methodenausbildung und in der empirischen Sozialforschung statt. Allerdings kommt es hierbei nicht zu einem grundlegenden Verständnis von Theoriearbeit oder von Theoriebildung, das über die eine Generalisierung von konkreten empirischen Beobachtungen hinausgeht. Dies führt

\footnotetext{
${ }^{6}$ Martin (2015: 1ff.) nutzt den Oberbegriff "theory-work“ und subsumiert darunter folgende sechs Theoriebegriffe: 1) Theory-Ology, 2) Theory as Canonical and Important Propositions, 3) Theory as Generalization, 4) Theory as Vocabulary, 5) Theory as Critique und 6) Theory-Work as Orthologics.

${ }^{7}$ Hier kritisiert Martin (2015: 3) vor allem das immer populärer werdende Genre der Zeitdiagnose. Er schreibt dazu: „With the rejection of theory-ology, theory increasingly meant, empirical generalizations', and so interest shifted to those who made wild overgeneralizations, sometimes peppered with poorly developed neologisms and references to well-known vocabularies in which these neologisms could not possibly survive."

${ }^{8}$ In der Regel werden angehende Soziolog*innen in eine bestimmte theoretische Schule oder in einen speziellen Forschungsbereich hineinsozialisiert, dabei werden mehr oder weniger implizit die Standards und Kriterien der jeweiligen Schule übernommen.
} 
unseres Erachtens dazu, dass die allgemeine Theoriedebatte und die Ansätze empirischer Theoriebildung relativ unverbunden nebeneinanderstehen.

\section{Die Standards empirischer Theoriebildung und Swedbergs theorizing-Konzeption}

In einschlägigen Lehrbüchern zu Methoden der empirischen Sozialforschung finden sich nicht nur methodische Anleitungen und Erläuterungen zur Strukturierung von Forschungsprozessen, sondern es werden zumeist auch Grundlagen der Wissenschaftstheorie vermittelt und nicht zuletzt auch Kriterien und explizite Handlungsanleitungen zur Theoriebildung (vgl. Atteslander 2010: 22ff.; Schnell et al. 2011: 45ff.; Kromrey et al. 2016: 46ff.; Diekmann 2018: 140ff.). Dargestellt werden insbesondere Regeln der Logik (wie interne Konsistenz, Widerspruchsfreiheit und formale Adäquanz), grundlegende Forschungsstrategien im Verhältnis von Theorie und Empirie (vor allem die Unterscheidung von Induktion und Deduktion) aber auch basale Charakteristika, guter' Theoriebildung. Genannt werden hier neben Widerspruchsfreiheit und interner Konsistenz unter anderem auch Kriterien wie Einfachheit, Überprüfbarkeit, Erklärungsfähigkeit und nicht zuletzt auch Generalisier- und Falsifizierbarkeit, die es bei der Erstellung von Theorien zu berücksichtigen gelte. Theorien und Theoreme, die diesen Kriterien nicht genügen, werden hier in der Regel als unzureichend abgelehnt.

Auffällig ist jedoch, dass das Theoretisieren in den Methodenlehrbüchern hauptsächlich mit Hypothesenbildung und mit ,empirischer Theorie' gleichgesetzt wird. Theorien werden daher ganz im Sinne von Abends erstgenanntem Theorieverständnis (theory ${ }_{1}$ ) vor allem als ein „System von Hypothesen über einen Gegenstandsbereich" definiert (Kromrey et al. 2016: 47f.), die in einer „logischen“ und „widerspruchsfreien“ Art und Weise miteinander zusammenhängen (sollen). Eine darüber hinaus gehende Verbindung zur generellen Theoriediskussion und zur (Weiter-)Entwicklung allgemeiner soziologischer Theorie(n) wird jedoch üblicherweise nicht hergestellt. Allgemeine soziologische Theorien werden lediglich als Ausgangspunkt und Hintergrundfolie des Forschungsprozesses oder als grundlegende „begriffliche Bezugssysteme“ und „(Denk)Werkzeuge“ eingeführt (Kromrey et al. 2016: 52). Das Verhältnis von empirischer Sozialforschung und allgemeiner Theorie sowie auch die Rolle von allgemeineren theoretischen Konzeptionen im Forschungsprozess bleibt eigentümlich unspezifisch und unterbestimmt. ${ }^{9}$

\footnotetext{
${ }^{9}$ Für Schnell et al. (2011: 5) etwa besteht „[e]in großer Teil der Arbeit in der Phase der Theoriebildung aus der Literaturanalyse, also der Sichtung und Bewertung der zum Thema existierenden Fachliteratur". Es wird zudem bedauert, dass die meisten Theorien in den Sozialwissenschaften zu ungenau formuliert seien, sodass eine empirische Untersuchung eine Präzisierung der zur Erklärung verwendeten Konzepte
} 
Neben den soeben genannten Einführungen zur Methodenlehre, die in der Regel eine starke Orientierung an der Logik quantitativer Sozialforschung aufweisen, gibt es auch innerhalb der qualitativen Sozialforschung Ansätze, die sich explizit mit dem Problem der theoretischen Generalisierung beziehungsweise mit Theoriebildung auseinandersetzen. Als prominentes Beispiel ist hier vor allem die Grounded Theory hervorzuheben (Glaser/Strauss 1967; siehe auch Strauss 1998). Sie basiert auf einem induktiven Forschungsverfahren, das auf die Erstellung einer neuen Theorie über einen - durchaus begrenzten - Gegenstandsbereich abzielt. Allerdings wird ein Bezug zur allgemeinen Theoriediskussion hierbei explizit vermieden, weil dies der induktiven Forschungslogik widerspräche. Die Theorien aus der Grounded Theory bleiben immer phänomenbezogen und bieten daher kein allgemeines Modell des Theoretisierens. ${ }^{10}$

In diese Leerstelle stößt nun Richard Swedberg mit seinen aktuelleren Veröffentlichungen zum Prozess des theorizing in der Soziologie (vgl. Swedberg 2012b, 2014a, 2014b, 2016, 2017). Swedbergs Ausgangspunkt ist die Feststellung, dass die Reflexion über den Akt des Theoretisierens und über den Prozess der Theoriebildung in der Soziologie bisher kaum stattgefunden hat. Sein Ziel ist es daher, theorizing als eine eigenständige Dimension und Praxis soziologischer Theoriebildung zu etablieren und in der soziologischen Forschung und Lehre zu verankern. Theorizing bezeichnet nach Swedberg den Prozess der Theoriebildung beziehungsweise der Theorieproduktion, der im günstigsten Fall zur Entstehung einer neuen Theorie führt. ${ }^{11}$ Theorie und theorizing sind für Swedberg somit nicht identisch: „Before theory comes theorizing" (Swedberg 2016: 6), sodass theorizing buchstäblich als Theoriebildung verstanden werden soll.

Eine weitere zentrale Unterscheidung ist für Swedberg die Unterscheidung zwischen dem Prozess der Theoriebildung einerseits und der strengen wissenschaftlichen Prüfung und Bewertung von theoretischen Konzeptionen andererseits. Auf diese Weise möchte er die Theoriediskussion vom Ballast methodologischer Richtungsstreits befreien und den Prozess des Theoretisierens selbst in den Mittelpunkt stellen. Um diese Differenz zu plausibilisieren, greift

und Begriffe voraussetze (Schnell et al. 2011: 7). Weitergehende Ausführungen oder konkretere Anleitungen zur Praxis des Theoretisierens sind unter dem Stichwort "Theoriebildung" hier jedoch auch nicht zu finden.

${ }^{10}$ Zur allgemeinen Debatte über das Verhältnis von Theorie und qualitativer Sozialforschung siehe auch Kalthoff et al. 2008.

${ }^{11}$ Theorizing ist nach Swedberg (2014a: 101ff.) somit vor allem ein abduktives Verfahren im Sinne von Charles Sanders Pierce, das zur Entdeckung und Erklärung von Neuem angelegt ist. Aus Platzgründen müssen wir uns auf den konzeptionellen Kern von Swedbergs theorizing beschränken und können bestimmte Aspekte, wie den Bezug auf Pierce (siehe dazu vor allem Swedberg 2012a), nicht behandeln. 
Swedberg auf die von Popper (2005) und Reichenbach (1961) eingeführte Unterscheidung von context of discovery und context of justification zurück (Swedberg 2012b: 3, 2014a: 17f.), was im Deutschen üblicherweise mit der Unterscheidung von Entdeckungs- und Rechtfertigungszusammenhang wiedergeben wird. Ersterer ist als kreativer Prozess zu verstehen, in dem Neues entdeckt wird, letzterer als wissenschaftlich-methodisch korrekte Ausarbeitung und Darlegung des Neuentdeckten nach Kriterien, wie sie etwa in Methodenbüchern und in den Grundlagen zur Wissenschaftstheorie vermittelt werden.

Das theorizing verortet Swedberg ausschließlich im Entdeckungszusammenhang. Das Endprodukt des theorizing, die aufgestellte Theorie, muss am Ende jedoch den strengen wissenschaftlichen Kriterien im Rechtfertigungszusammenhang Rechnung tragen (Swedberg 2012b: 14f.). Um Erklärungen - und damit in seinem Sinne, Theorien' - zu produzieren, entwirft Swedberg ein vierstufiges Programm, das das theorizing mehr oder weniger systematisch anleiten soll: (1) Der erste und wichtigste Schritt ist für Swedberg die Beobachtung von etwas Neuem, Problematischem oder Überraschendem (Swedberg 2014a: 29ff.), kurzum: ein Rätsel beziehungsweise ein „Puzzle“, für das spontan keine Erklärung gefunden werden kann (Swedberg 2014a: 37ff.). Theorizing weist nach Swedberg - ähnlich wie bei den Anleitungen zur empirischen Methodenlehre - immer einen konkreten Bezug zur Empirie beziehungsweise zu einem sozialen Phänomen auf. Da das theorizing in Gänze im Entdeckungszusammenhang verortet wird, sollte der erste Schritt der Beobachtung laut Swedberg nicht zu stark an methodische Restriktionen gebunden sein. Im Modus der Entdeckung sollen neben eigenen Beobachtungen möglichst vielfältige Arten von Informations- und Inspirationsquellen herangezogen werden, wie etwa Zeitungsberichte, Bücher, aber auch Anekdoten und Tagträume - frei nach der Devise: „Anything goes!“ (Swedberg 2012b: 11; Hervorhebung im Original)

Die weiteren drei Schritte des theorizing sind (2) die Benennung, Konzeptionalisierung und Typologisierung der empirischen Beobachtung, (3) die Suche nach Mustern, Analogien und Metaphern und (4) die Formulierung einer schlüssigen Erklärung (Swedberg 2014a: 52ff.). Swedberg zählt hierbei auch eine Reihe von Strategien und Praktiken auf, die das theorizing anregen und verbessern sollen - etwa der Rückgriff auf Experimente, Vergleiche und kontrafaktischem Denken als Heuristiken („heuristic tools") bei der Plausibilisierung von 
Erklärungen. ${ }^{12}$ Entscheidend ist jedoch, dass der Prozess des theorizing nach Swedberg ohne den Akt des Erklärens nicht abgeschlossen ist.

Mit seiner Konzeption des theorizing legt Swedberg somit einen Ansatz vor, mit dem gegenstandsbezogene Erklärungen generiert werden können. Ihm gebührt durchaus Verdienst dafür, das Thema Theoriebildung überhaupt (wieder) auf die Agenda gesetzt zu haben. Darüber hinaus regt Swedbergs Ansatz auch dazu an, neu über das Verhältnis von theoretischer Reflexion und empirischer Forschung nachzudenken. Denn gerade durch die Trennung von Entdeckungsund Rechtfertigungszusammenhang gelingt es Swedberg, die kreativen und schöpferischen Aspekte theoretischer Reflexionen zu betonen. ${ }^{13}$ Seine methodische Anleitung zum theorizing (im Sinne von Theoriebildung) ist unseres Erachtens jedoch in keiner Weise dazu geeignet, als allgemeines Modell für Theoriebildung und Theoriearbeit in der Soziologie zu dienen. Denn wir bezweifeln grundsätzlich, dass Swedbergs theorizing-Konzeption der Komplexität der Theoriebildung und der Praxis des Theoretisierens gerecht wird. Swedbergs Vorschlag greift schlichtweg zu kurz, wenn er den Prozess des Theoretisierens auf einen schöpferischen und kreativen Akt im Modus des Entdeckungszusammenhangs reduziert. Die Arbeit an und mit Theorien verlangt in der Regel weitaus mehr als eine möglichst ungezügelte kreative Energie.

Es ist zudem zu bezweifeln, dass sich das Theoretisieren tatsächlich immer auf die Schaffung einer neuen Theorie beziehungsweise auf die Erstellung von Abduktionen reduzieren lässt. Viele etablierte Formen des Forschens oder auch viele weitere Aspekte des Theoretisierens werden hier systematisch ausgeblendet und gar nicht als Teil der Praxis des Theoretisierens angesehen. Swedberg verkürzt sein theorizing auf ein Theorieverständnis, das trotz aller Offenheit für unvoreingenommene theoretische Inspiration am Ende doch sehr stark an die rigiden wissenschaftstheoretischen Grundsätze der erklärenden Soziologie angelehnt ist (vgl. Swedberg 2014a: 98ff.). Für ein tieferes Verständnis der Praxis des Theoretisierens ist das ein gravierendes Problem.

\section{Die Modi soziologischer Theoriebildung: Zur Vielfalt des Theoretisierens in der Soziologie}

\footnotetext{
12 Swedberg nennt auch eine ganze Reihe von unterschiedlichen Erklärungsarten und Erklärungsstrategien. Explizit unterscheidet er in seinen Texten teleologische, funktionale, vergleichende und kontrafaktische Erklärungsstrategien (Swedberg 2014a: 106ff.).

${ }^{13}$ Neben einer grundsätzlich positiven Aufnahme des theorizing (siehe zum Beispiel die Beiträge in Swedberg 2014b; Krause 2016; Silver 2019) gibt es aber auch äußerst kritische Stimmen (vgl. insbesondere Anicker 2019; Weiß 2017).
} 
Obwohl die soeben vorgestellten Ansätze die Bedeutung von Theoriearbeit im empirischen Forschungsprozess betonen, verhindern sie doch zugleich ein grundlegendes Verständnis der Rolle des Theoretisierens in der Soziologie. ${ }^{14}$ Versteht man den Prozess des Theoretisierens beziehungsweise die Theoriearbeit lediglich als eine Methode zur Erfassung und Systematisierung von empirischen Beobachtungen, so mag dies als eine - sehr enggeführte Bestimmung von Theoriearbeit genügen. Begreift man den Akt des Theoretisierens jedoch als eine vielschichtige Praxis theoretischer Generalisierung und als begriffliche Reflexion einer Vielzahl von unterschiedlichen empirischen und auch nicht unmittelbar empirisch zugänglichen Sachverhalten, ergibt sich ein ganz anderes Bild.

Es unserer Ansicht nach notwendig, sich bei der Reflexion über Theoriebildung in der Soziologie vor allem die unterschiedlichen Bezugsprobleme und Geltungsansprüche des Theoretisierens bewusst zu machen. Zudem ist jede Art von soziologischer Reflexion, ob sie nun stark empirisch angeleitet ist oder sich vorwiegend aus der Auseinandersetzung mit Theorien ableitet, in einem gemeinsamen Korridor wissenschaftlicher Reflexion zu verorten (vgl. Alexander 1982: 1ff.). ,Theorie' und ,Empirie' existieren nicht unabhängig voneinander, sie sind vielfach miteinander verwoben. Es gibt jedoch gravierende Unterschiede zwischen den Abstraktionsniveaus von Theorien und ihren empirischen Geltungsansprüchen. Und es gibt allgemeine Theorien beziehungsweise erkenntnisleitende Vorannahmen (sog. allgemeine Propositionen oder Metatheorien), auf die die unabhängig von unmittelbaren empirischen Bezügen Bezug genommen wird. All dies gilt es bei einer Systematisierung von Theoretisierungspraktiken in der Soziologie zu berücksichtigen. Auf Basis dieser Annahmen arbeiten wir nachfolgend zentrale Elemente und Modi des Theoretisierens heraus, die sich nicht auf einen einzigen Begriff von ,Theoriearbeit' und, Theoriebildung'verengen lassen.

\subsection{Zum Verhältnis von ,Theorie' und ,Empirie': Unterschiedliche Elemente und Grade theoretischer Abstraktion}

Es ist für ein grundlegendes Verständnis der Rolle von Theorie in der wissenschaftlichen Praxis wichtig anzuerkennen, dass Theorie und Empirie keine getrennten Welten darstellen, sondern

\footnotetext{
${ }^{14}$ Um Missverständnissen vorzubeugen: Nichts spricht gegen empirisch gestützte Theoriebildung oder gegen die Überprüfung, Validierung und (Weiter-)Entwicklung von Theorien anhand von empirischer Forschung. Dies ist eine fundamentale wissenschaftliche Praxis. Zu bezweifeln und vollends abzulehnen ist jedoch die Vorstellung, dass dies die einzige und die einzig richtige Art der soziologischen Theoriebildung und Erkenntnis sei.
} 
stets wechselseitig aufeinander bezogen sind. Spätestens mit Kants Grundsteinlegung der modernen Erkenntnistheorie wird die Annahme weithin geteilt und anerkannt, dass es keine theoriefreie empirische Beobachtung und auch keine empirielose Theorie gibt (vgl. Kant 1998). Diese erkenntnistheoretische Grundannahme wurde vom Positivismus im 19. Jahrhundert zwar immer wieder in Frage gestellt. Heute gibt es in der Wissenschaft aber kaum jemand, der die kruden erkenntistheoretischen Prämissen des klassischen Positivismus noch ernsthaft teilt, auch der Kritische Rationalismus geht von einer starken Verschränkung von Theorien und Beobachtungen aus. ${ }^{15}$

Jeffrey Alexander (1982: 1ff.) hat diese erkenntnistheoretischen Grundlagen im ersten Teil seiner publizierten Vorlesung Theoretical Logic in Sociology sehr anschaulich auf die Soziologie übertragen (vgl. hierzu auch Joas/Knöbl 2004: 24ff.). Nach Alexander changieren die wissenschaftlichen Erkenntnisstrategien zwischen den beiden Extrempolen der ,metaphysischen' und der ,empirischen' (Um-)Welt. Beide Bereiche sind - dies muss betont werden - in ihrer reinen Ausprägung nicht zugänglich. Sie bilden folglich (je unerreichbare) Umwelt und Bezugspunkte wissenschaftlichen Denkens und Arbeitens, sowohl der kontrollierten empirischen Beobachtung als auch der theoretischen Abstraktion. Alexander geht somit von der Prämisse aus, dass ein direktes Abbild der empirischen Wirklichkeit nicht möglich ist, da unsere Beobachtungen, unsere Sicht auf die Welt auf vielfältige Weise mit methodologischen Grundannahmen, Definitionen, theoretischen Kategorien und Modellen durchsetzt ist. Diese entspringen der theoretischen Reflexion und liegen damit näher an der metaphysischen Umwelt. Theoretisch bedingte Beobachtungen sind folglich die nahest mögliche Annäherung an die empirische Welt. In dieser Weise sind theoretische Abstraktionen und empirische Beobachtungen vielfältig miteinander verknüpft, und die verschiedenen Elemente theoretischer Abstraktion lassen sich gradueller in einem „Kontinuum wissenschaftlicher Abstraktion" verorten (siehe Abbildung 2).

\footnotetext{
${ }^{15}$ Auf die Verschränkung und Untrennbarkeit von theoretischen Begriffen und empirischer Beobachtung wurde in der Philosophiegeschichte von Kant bis Popper vielfach und immer wieder aufs Neue hingewiesen (Skirbekk 1977). Und selbst Popper, der sich für eine unabhängige empirische Überprüfung von Theorien an der Empirie stark macht, konstatiert: „Es gibt keine reinen Beobachtungen: sie sind mit Theorien durchsetzt und werden von Problemen und von Theorien geleitet" (Popper 2005: 89). Dies heißt nichts anderes als dass die Begriffe (Theorien), mit denen wir die empirische Welt wahrnehmen, der empirischen Beobachtung vorausgehen (vgl. Popper 1984). Theorien prägen unser Denken und sie strukturieren unsere Wahrnehmung. Es ist also nicht unerheblich, mit welchen Begriffen beziehungsweise Theorien wir die empirische Welt beobachten.
} 
Neben der vielschichtigen Verschränkung von Theorie und Empirie liefert Alexander eine weitere, für ein Grundverständnis soziologischer Theoriearbeit wichtige Differenzierung. Er zeigt, dass es keine einheitliche Praxis beziehungsweise keinen einheitlichen Akt des Theoretisierens gibt, sondern dass theoretische Reflexion im konkreten Fall sehr unterschiedliche Aspekte umfasst - etwa durch Bezugnahme auf allgemeine Vorannahmen, Konzepte, Definitionen und Klassifikationen, oder durch die Erstellungen von Modellen und mehr oder weniger komplexen Allgemeinaussagen. All diese Bestandteile werden in der Regel mit Theorie(n) assoziiert und sind in diesem Sinne wesentliche Bestandteile der Praxis theoretischer Generalisierung. Es gibt allgemeine Theorien, die eine Vielzahl dieser Elemente in sich vereinen, dies sind in der Regel die Sozial- und Gesellschaftstheorien. Es gibt theoretische Überlegungen und Bezugnahmen auf theoretische Konzeptionen, die nur einige wenige der vorgenannten Elemente beinhalten - etwa, wenn Begriffe definiert werden oder auf etablierte theoretische Begriffe und Klassifikationen zurückgriffen wird und diese verfeinert und weiterentwickelt werden. Die Grundelemente theoretischer Reflexion sind somit immer die gleichen, was sich jedoch ändert, sind die Ziele, Bezugspunkte und Abstraktionsebenen. Dies wird nachfolgend genauer erläutert.

\subsection{Unterschiedliche Bezugsprobleme und Abstraktionsniveaus von soziologischen Theorien}

Wir haben im zweiten Kapitel bereits herausgearbeitet, dass es in der Soziologie sehr unterschiedliche Theoriebegriffe gibt, die sich mit unterschiedlichen wissenschaftstheoretischen und methodologischen Standpunkten verbinden. Diese verschiedenen Standpunkte und Grundpositionen implizieren auch unterschiedliche Vorstellungen von Theoriearbeit und damit auch unterschiedliche Theoretisierungspraktiken. Es gibt in der Soziologie auch eine Vielzahl unterschiedlicher Anwendungsfelder, aus denen sich verschiedene Theorien mit recht unterschiedlichen Bezugsproblemen ergeben. Häufig werden soziologische Theorien daher nach ihrem zentralen Gegenstand differenziert und daher zum Beispiel als Konflikt-, Handlungs- oder Austauschtheorien bezeichnet, oder sie werden nach Geltungsbereichen gegliedert (etwa als Handlungs- und Strukturtheorien, Sozial- und Gesellschaftstheorien oder als Mikro-, Meso- und Makrotheorien). Auch dies hat Auswirkungen auf die Art des Theoriebezugs und damit auch auf den Charakter des Theoretisierens. 
Viel wichtiger als die methodologische und inhaltliche Unterscheidung von Theorien erscheint uns im Hinblick auf die Reflexion unterschiedlicher Modi des Theoretisierens jedoch eine Differenzierung von Theorien nach ihrer Reichweite beziehungsweise nach ihren Abstraktionsniveaus. Es macht, um dies nur an einem Beispiel zu verdeutlichen, einen wesentlichen Unterschied, ob eine Theorie auf die Erklärung eines ganz spezifischen empirischen Zusammenhangs (etwa in Gestalt eines Modells oder einer zu testenden Hypothese) abzielt oder ob ein allgemeines Theorem (etwa die Evolutionstheorie) zur Deutung und Erklärung eines größeren gesellschaftlichen Entwicklungsprozesses heranzogen wird. Wenn es also um Theoriebildung geht, ist ein grundlegender Faktor das Abstraktionsniveau der zu bildenden Theorie.

Auf die Unterschiede in der Reichweite und Bezugssysteme soziologischer Theorien wurde in der Literatur zwar schon mehrfach hingewiesen (vgl. Haller 1999: 41; Turner 1986: 17ff.), allerdings werden diese Unterschiede in der Reflexion über das Theoretisieren zu wenig berücksichtigt. Auch die eingangs bereits erwähnte semantische Unterscheidung von Abend (2008) weist ganz deutlich auf unterschiedliche Bezugsprobleme und Abstraktionsniveaus von Theorien hin, die er in seinem Aufsatz jedoch nur wenig expliziert. Wenn man die semantische Unterscheidung jedoch ernst nimmt, so implizieren die genannten Theoriebegriffe auch sehr unterschiedliche Standards und Praktiken der Theoriebildung, die es zu explizieren gilt.

Die Abstraktionsgrade von Theorien werden üblicherweise wie folgt bestimmt: Auf der ersten Abstraktionsstufe finden sich „gegenstandsbezogene empirische Generalisierungen“, darauf folgen "Theorien mittlerer Reichweite" und "formale Modelle" und den Abschluss bilden „axiomatischen Theorien“ mit dem höchsten Grad an Abstraktion (vgl. Turner 1986: 17ff.). ${ }^{16}$ Wir orientieren uns bei unserer Unterscheidung zentraler Modi soziologischer Theoriebildung an dieser grundlegenden Systematik. Zugleich müssen aber auch einige weitere Aspekte berücksichtigt werden, die bei der Unterscheidung der verschiedenen Bedeutungsebenen immer wieder genannt werden: Dies sind die eigenständige Bedeutung von kritischen Theorien

\footnotetext{
${ }^{16}$ In Anlehnung an den Wissenschaftstheoretiker Mario Bunge (1996) unterscheidet Max Haller (1999: 41) Theorien in der Soziologie ebenso nach ihrem Geltungsbereich: a) spezielle Theorien oder Modelle, die gegenstandsbezogen sind, b) allgemeine Theorien mit universellerem Geltungsanspruch (Haller nennt hier als Beispiele etwa die Bezugsgruppentheorie oder die Keynesianische Wirtschaftstheorie), c) Supertheorien (,hypergeneral theories") wie die Evolutionstheorie oder andere übergreifende Theorien und d) Gerüsttheorien ("scaffolding theories"), die in der Regel noch allgemeiner formuliert sind als Supertheorien, da sie generelle Propositionen setzen, die nicht direkt empirisch überprüfbar sind. Als Beispiele werden hier etwa die allgemeine Systemtheorie oder auch die Rational-Choice-Theorie genannt.
} 
und normativer Theoriebildung (theory6) sowie die Vorstellung von Theoriearbeit als Diskussion grundlegender Axiome und Vorannahmen (theory7) (Abend 2008; vgl. Abbildung 1). Auf Basis dieser Vorüberlegungen lassen sich nun unseres Erachtens einige zentrale Modi soziologischer Theoriebildung herausarbeiten, mit denen sich jeweils spezifische Standards und Praktiken des Theoretisierens verbinden.

\subsection{Sechs zentrale Modi soziologischer Theoriebildung}

Auf Basis der vorangegangenen Vorüberlegungen lassen sich insgesamt sechs Modi von Theoriebildung unterscheiden, die die soziologische Forschungspraxis grundlegend prägen ganz gleich ob sie im Einzelfall eher auf die empirische oder theoretische Umwelt hin ausgerichtet ist (s. Abbildung 3). Jegliches Theoretisieren in der Soziologie ist daher sowohl von der Reichweite der Theorie als auch vom Theorie-Empirie-Nexus her zu denken. Bei den sechs zentralen Modi, die wir auf unterschiedlichen Ebenen der Generalisierung im Theorie-EmpirieKontinuum verorten, weisen einige der Modi einen stärkeren Empiriebezug auf beziehungsweise sind stärker mit empirischer Beobachtung verbunden. Andere wiederum sind eher in im Bereich (meta-)theoretischer Reflexion angesiedelt. Die Art des Theoriebezugs hängt somit entscheidend vom Ziel der wissenschaftlichen Forschung ab, und dieses Ziel bestimmt den jeweiligen Modus des Theoretisierens. Wir unterscheiden folgende sechs Modi des Theoretisierens:

(1) Theoretisierung zur Erklärung oder zum Verständnis eines konkreten empirischen Problems beziehungsweise Phänomens (etwa eines festgestellten Zusammenhangs zwischen zwei Ereignissen oder einer fraglichen Konstellation von Ereignissen);

(2) theoretische Generalisierungen in speziellen Anwendungsfeldern und Teilbereichen der Soziologie, also die Arbeit an und mit Theorien begrenzter Reichweite oder speziellen Theorien;

(3) die kritische Theoriebildung beziehungsweise die Arbeit mit und an normativen Theorien;

(4) die Arbeit an ausgewählten Grundproblemen der Sozial- und Gesellschaftstheorie;

(5) Theoriearbeit im Kontext allgemeiner soziologischer Theorie, d. h. die Reflexion allgemeinerer sozial- und gesellschaftstheoretischer Grundprobleme und die allgemeine soziologische Theoriebildung und 
(6) die Reflexion grundlegender meta- und wissenschaftstheoretischer Probleme und Fragestellungen.

Wir behaupten selbstverständlich nicht, dass diese Modi des Theoretisierens in der soziologischen Praxis stets in Reinform vorkommen; es gibt unzählige Überlappungen und auch Mischtypen. Auch nehmen wir, obwohl wir Abstraktionsebenen unterscheiden, keine implizite Priorisierung oder Hierarchisierung vor. Es geht also nicht darum zu behaupten, dass Theoriearbeit umso besser ist, je abstrakter sie ansetzt. Vielmehr wollen wir zeigen, dass das Theoretisieren nicht auf der ersten Stufe aufhört - wie es etwa bei Swedberg der Fall ist. Die einzelnen Stufen des Theoretisierens weisen jeweils spezifische Bezüge zur Theorie und zur Empirie auf, sodass ein wesentlicher Akzent der nachfolgenden Darstellung auf der Verortung im Theorie-Empirie-Kontinuum und auf der Darstellung typischer Arten des Theoretisierens auf jeder Ebene von Theoriebildung liegt. Dies ist für uns einer der zentralen Aspekte bei der Reflexion über die Praxis des Theoretisierens. Es folgt eine genauere Darstellung und Erläuterung der einzelnen Modi, bei der wir insbesondere auch auf wichtige Standards und konkrete Praktiken eingehen, die sich mit den einzelnen Modi des Theoretisierens verbinden.

\section{Abbildung 3 ungefähr hier}

Modus 1-Erklärung konkreter empirischer Phänomene: Dieser Modus der Theoretisierens ist am stärksten mit dem Anspruch einer möglichst umfassenden empirischen Fundierung verknüpft, denn es geht zentral um die soziologische Erklärung sozialer Phänomene, die auch als „Rätsel“ konzipiert werden. Sie entspricht am ehesten der Praxis der Theoriebildung, wie sie in Lehrbüchern zur empirischen Sozialforschung dargestellt und propagiert wird (vgl. Kapitel 3). Dabei ist die Suche nach Erklärungen für empirische Phänomene nicht durch eine bestimmte Methode oder Methodologie determiniert. Es finden sich hier gleichermaßen quantitative wie qualitative, induktive wie deduktive Forschungsverfahren und viele Kombinationen und Mischformen. Stark dominiert wird diese Art der Theoriebildung jedoch von den Konzepten und Methodologien der ,analytischen' beziehungsweise, erklärenden' Soziologie, wobei Theoriebildung weitgehend mit Hypothesenbildung gleichgesetzt und assoziiert wird. Ziel ist eine möglichst exakte Abbildung und "Modellierung" empirischer Beobachtungen und Zusammenhänge („Korrelationen“) (Braun 2008) - bevorzugt auch mithilfe einer 
mathematischen Syntax. Es gibt in den Reihen der analytischen und erklärenden Soziologie jedoch auch Verfechter*innen von weniger rigorosen Anforderungen an theoretische Exaktheit - Swedbergs Konzeption von theorizing und die Soziologie sozialer Mechanismen in der Tradition von Merton bis Hedström wären hier prominente Beispiele (siehe für einen Überblick zu Mechanismen Hedström/Ylikoski 2010).

Eine sehr übliche Art des Umgangs mit Theorie(n) in der empirischen Forschung ist auch die Verwendung von Theorien als Heuristiken. Hier geht es weniger um Theoriebildung, sondern in deduktiver Logik um einen theoretisch informierten und kontrollierten Zugang zum empirischen Forschungsobjekt. Häufig werden hierbei Grundbegriffe, Definitionen, Klassifikationen und umfassendere Konzepte von bereits bestehenden theoretischen Ansätzen als Grundlage und Ausgangspunkt eigenen Forschens herangezogen. Heuristiken dienen somit einerseits der Strukturierung von Forschungsdesigns, sodass ausgehend von Definitionen, Klassifikationen und allgemeineren Konzeptionen überhaupt erst in den Blick genommen werden kann, um was bei der empirischen Forschung eigentlich geht. Theorien als Heuristiken werden andererseits auch bei der Interpretation, d. h. beim Verstehen und Erklären, von empirischen Beobachtungen und Zusammenhängen verwendet. Heuristiken prägen demnach ganz entscheidend die Wahrnehmung und Deutung sozialer Wirklichkeit. Häufig sind Innovationen in der soziologischen Forschung und Theoriebildung von der Umstellung von Heuristiken abhängig. Abbott (2004) bezeichnet diese als "heuristic moves" und widmet ein ganzes Lehrbuch der Reflexion der Bedeutung von Heuristiken in der soziologischen Forschungspraxis. Auch Swedberg nimmt in seinem theorizing-Konzept explizit Bezug auf die Rolle von Konzepten als Heuristiken und auf die produktive Funktion von heuristic moves, gerade im Hinblick auf die Produktion von neuen und originellen Erkenntnissen und Erklärungen (Swedberg 2014a: 58ff., 134ff.).

Vernachlässigt wird häufig jedoch, dass auch die Grounded Theory nach Glaser und Strauss (1967; siehe auch Strauss 1998) einen Erklärungsanspruch impliziert und somit ebenso in diesen Modus des Theoretisierens einzuordnen ist. Ganz im Gegensatz zum Forschen mit Heuristiken ist die Grounded Theory ein induktives Forschungsverfahren beziehungsweise eine Methodologie zur empirisch gestützten Generierung von Theorien, das sich vor allem auf die systematische Sammlung und Auswertung qualitativer Daten stützt.

Da sich so unterschiedliche Ansätze wie die Grounded Theory, Swedbergs theorizing und die „erklärende Soziologie“ hier verorten lassen, dominiert dieser Modus des Theoretisierens weder 
eine Methodologie noch ein einziger Erklärungsweg. Er orientiert sich also nicht an der herkömmlichen Trennung von qualitativer und quantitativer Sozialforschung, sondern dieser Modus weist den stärksten Bezug zur empirischen Umwelt auf und ist in diesem Sinne der Konkreteste. Anderseits ist zu beachten, wie stark grundlegende sozialtheoretische Annahmen und Konzeptionen bereits in die Basisannahmen der empirischen Forschung einfließen. Die empirische Forschung ist somit - ganz im Sinne Alexanders (1987) - stets durch tiefere Schichten der Theoriebildung mitbestimmt.

Modus 2 - Die Arbeit an Theorien begrenzter Reichweite und speziellen Theorien: Theorien begrenzter Reichweite ${ }^{17}$ oder spezielle Theorien sind in der Regel ebenso stark verknüpft mit empirischen Forschungsgegenständen und empirischer Forschung wie die Theoriebildung im erstgenannten Modus. Häufig gehen beide Modi der Theoriearbeit auch ineinander über: dann etwa, wenn spezielle Theorien bei der Erklärung eines empirischen Phänomens herangezogen werden oder wenn Erkenntnisse aus der Forschung zu einem sehr begrenzten Sachverhalt gebündelt werden und in eine generellere Theorie über einen bestimmten soziologischen Gegenstand übertragen werden. Theorien begrenzter Reichweite sind Theorien zu spezifischen Gegenständen wie Organisationen, Gruppen, Familien oder zu gesellschaftlichen Teilbereichen wie etwa Kunst, Wissenschaft, Politik usw. Zu allen diesen Gegenständen und Bereichen gibt es „spezielle Theorien“ und spezielle Standards der Theoriebildung, die die Diskussionen in den empirischen Forschungsfeldern prägen und anleiten und die wiederum von empirischen Forschungsergebnissen beeinflusst werden. Spezielle Theorien haben somit in der Regel keinen umfassenden Deutungs- und Erklärungsanspruch, sondern die Theoriebildung findet in engem Austausch mit empirischer Forschung statt.

Theoriebildende Forschung umfasst in diesem Modus ein Bündel von unterschiedlichen Strategien. Sie ist erstens, wie bereits beschrieben, sensibel gegenüber neuen empirischen Erkenntnissen, Insofern werden empirische Forschungen, sowohl quantitative als auch qualitative, wertschätzend berücksichtigt. Zweitens orientiert sich die Theoriearbeit auch an bereits bestehenden Theorien begrenzter Reichweite. Theorien des Politischen müssen beispielsweise bereits erlangte (theoretische) Erkenntnisse der Politischen Soziologie

\footnotetext{
${ }^{17}$ Wir folgen hier der Typologie von Haller (1999). Aber auch Gesa Lindemann (2008: 109) spricht von "Theorien begrenzter Reichweite“ im Gegensatz zu Mertons (1995) Bezeichnung von „Theorien mittlerer Reichweite", um den Gegenstandsbezug zu verdeutlichen und eine Hierarchisierung zu vermeiden.
} 
berücksichtigen und sich zu ihnen positionieren. Drittens wird die Forschung oftmals von etablierten, allgemeineren Theorien (Paradigmen) beeinflusst. Die starke empirische Fundierung und Einschränkung des Geltungsbereichs spezieller Theorien bedeutet somit nicht, dass diese nicht grundlegend durch allgemeine Sozial- und Gesellschaftstheorien mitgeprägt sind. Im Gegenteil: Häufig sind auch die speziellen Theorien von Annahmen, Propositionen und Theoremen der „Klassiker“ und der allgemeinen Theoriediskussion mitgeprägt. Zu erwähnen wären hier etwa vielfältige ressourcenbasierte Ansätze (in Anlehnung an Marx) in diversen speziellen Soziologien oder Anleihen aus den Grundbegriffen Max Webers oder der Luhmann'schen Systemtheorie. Auch spezielle Theorien werden somit nicht ausschließlich „empirisch“ formuliert, sondern sind zugleich vielfältig begrifflich-theoretisch strukturiert. Theorien begrenzter Reichweite und spezielle Theorien sind ebenso stark auf die empirische Umwelt hin bezogen wie der erste Modus, gleichwohl zeichnen sie sich durch einen etwas höheren Geltungsanspruch aus beziehungsweise sie beziehen sich auf einen größeren Teilaspekt der sozialen Wirklichkeit.

Modus 3: Kritische und normative Theoriebildung: Kritisches Theoretisieren zielt darauf ab, auf Probleme oder Missstände in der sozialen Welt hinzuweisen beziehungsweise kritische Entwicklungen mithilfe theoretisch hergeleiteter Kriterien und Standards zu problematisieren. Hierbei wird in der Regel auf Anleihen von allgemeineren Theoremen aus der Gesellschaftstheorie und aus der (Sozial-)Philosophie zurückgegriffen, um normative Theorien zu bilden. „Normative Theoriebildung" ist in diesem Sinne als eine eigenständigen Bereich von Theoriebildung in der Soziologie anzusehen (vgl. hierzu auch Abend 2008: 180). Die kritischen beziehungsweise normativen Theorien stehen unseres Erachtens genau zwischen empirischer Forschung und allgemeiner theoretischer Reflexion. Der Hauptfokus liegt nicht auf der Erstellung einer möglichst neutralen, unvoreingenommenen Erklärung von allgemeinen gesellschaftlichen Phänomenen, Problemen oder Entwicklungen, sondern auf der Aufdeckung, Deutung und Erklärung gesellschaftlicher Missstände und Fehlentwicklungen. Dies kann durchaus induktiv erfolgen, also auf Basis gezielter empirischer Beobachtungen. Geläufiger ist jedoch die allgemeinere Reflexion von vorhandenen Forschungsdaten und die Entwicklung spezifischer Theorien, die ganze neue Heuristiken zur Erfassung der empirischen Wirklichkeit bereitstellen. Die feministischen Theorien wären hier ein Beispiel (Villa 2009), aber auch die Debatte über soziale Anerkennung (Honneth 1994). Der gesellschaftskritische Impetus der Theorie des 
kommunikativen Handelns von Jürgen Habermas (1995) kann hier ebenso eingeordnet werden wie die klassischen Studien und Abhandlungen von Adorno, Horkheimer und anderen Vertretern der klassischen Frankfurter Schule. All diese Debatten und Theorien sind einerseits eingebettet in grundlegende sozialphilosophische sowie sozial- und gesellschaftstheoretische Debatten, andererseits regen diese Debatten und Konzepte häufig auch unzählige empirische Forschungsarbeiten und nicht zuletzt auch die Entstehung ganzer Forschungsfelder an - z. B. die unterschiedlichen Bereiche der Armuts-, Ungleichheits- und Exklusionsforschung oder aktuell die Forschungsansätze rund um Begriffe wie Postwachstum, Nachhaltigkeit und Konvivialismus, aber auch die postkolonialen Theorien.

Modus 4-Die Bearbeitung einzelner Problemstellungen der Sozial- und Gesellschaftstheorie: Mit der Arbeit an spezifischen sozial- und gesellschaftstheoretischen Problemstellungen verlassen wir auf Alexanders Empirie-Theorie-Kontinuum endgültig die primäre Verankerung in der empirischen Forschung. Dies heißt nicht, dass in diesem Bereich der Theoriebildung Empirie überhaupt keine Rolle spielt. Selbstverständlich gibt es auch bei der Arbeit einzelnen Problemen der Sozial- und Gesellschaftstheorie einen Bezug zur empirischen Wirklichkeit. Häufig wird zu einzelnen Konzepten und Grundsatzfragen auch empirisch geforscht - etwa wenn es um die Rolle von Emotionen in der Praxistheorie geht (Scheer 2012) oder die Erforschung der Verknüpfung von Mensch und Technik (Karafillidis 2017). Der Fokus- oder Bezugspunkt beziehungsweise die Inspirationsquelle des Forschens und Theoretisierens ist allerdings nicht eine konkrete empirische Fragestellung oder ein empirisches Rätsel, sondern hier steht ganz im Gegenteil die Arbeit an theoretischen Begriffen und Konzeptionen im Mittelpunkt. Leitend ist damit nicht die empirische, sondern die von Alexander definierte metaphysische Umwelt (Alexander 1982). Ein großer Teil der Forschungspraxis im Bereich der Arbeit an Problemstellungen der Sozial- und Gesellschaftstheorie ist somit durch die Rekonstruktion, Diskussion und Weiterentwicklung bestehender theoretischer Einsichten bestimmt. Zumeist fokussieren sich die Forscher*innen hier auf nur sehr wenige theoretische Konzeptionen - etwa auf Bourdieus Praxisbegriff oder Luhmanns Systemtheorie - und bemühen sich um eine Weiterentwicklung einzelner Konzepte und Begrifflichkeiten innerhalb der Theorie oder mit Bezug zu sich wandelnden gesellschaftlichen Konstellationen.

Viele Fragestellungen ergeben sich inhärent aus den Theorien selbst, in Form von ungelösten Problemen und Baustellen einzelner Sozial- und Gesellschaftstheorien. Diverse Probleme 
werden aber auch durch Neujustierungen und Verschiebungen, nicht selten auch durch Moden und turns im allgemeinen Theoriediskurs hervorgerufen. Man denke hier etwa an die Durchsetzung neuer Theorien und Betrachtungsweisen, die bestehende Paradigmen herausfordern (wie etwa die neueren Praxistheorien die klassische Phänomenologie oder die Handlungstheorien usw.). Die Logik der Theoriearbeit wird hier also ganz wesentlich durch die Logik von theoretischen Paradigmen bestimmt, wie sie klassisch von Kuhn (1976) beschrieben wurde, sodass das Theoretisieren hier stark in Paradigmen („Schulen“) organisiert ist. Die Beschäftigung mit Grundproblemen des eigenen Paradigmas dient somit immer auch der Aktualisierung und Weiterentwicklung und damit der Verteidigung und Erhaltung der eigenen Denkschule.

Modus 5 - Allgemeine sozial- und gesellschaftstheoretische Theoriebildung: Die allgemeine sozial- und gesellschaftstheoretische Theoriebildung ist der umfassendste und generellste Modus des Theoretisierens in der Soziologie. Er wird derzeit nur noch selten praktiziert und ist seit der Zeit der, großen Theorien' - wohl auch verursacht durch grundlegende Verschiebung in der Logik soziologischer Forschung - immer mehr in den Hintergrund gerückt. ${ }^{18}$ Nicht viele Soziolog*innen können heute von sich behaupten, dass sie aktiv auf die Ausarbeitung einer Sozial- oder einer Gesellschaftstheorie abzielen. Sondern viele Theoretiker*innen arbeiten eher an ausgewählten Problemstellungen der aktuellen Sozial- und Gesellschaftstheorie (Modus 5), oder sie betreiben Theorieexegese, indem sie klassische Theorien und Fragestellungen neu strukturieren und in aktualisierter Form aufbereiten. Dies ist weder abwertend noch kritisch gemeint, denn auch diese Praktiken sind wichtige Bestandteile der Praxis von Theoriearbeit (Martin 2015: 10) - sie bilden häufig den Ausgangspunkt und die Grundlage für neue Theoriebildung und die Ausformulierung neuer Sozial- und Gesellschaftstheorien (vgl. Habermas' (1995) Zusammenführung pragmatischer und lebensweltlicher Ansätze zur Theorie kommunikativen Handelns).

Sozial- und Gesellschaftstheorien werden üblicherweise nicht gleichgesetzt, denn die eine Theorieart wird zumeist über die andere gesetzt. ${ }^{19}$ Im Sinne unserer Unterscheidung von Modi

\footnotetext{
${ }^{18}$ Zu vermuten wäre auch, dass die postkoloniale Kritik an bestehenden Theorien dazu geführt hat, dass hegemoniale Positionen in der Soziologie heute schwerer durchzusetzen sind, sodass "Großtheoretiker" wie Parsons, Luhmann oder auch Bourdieu heute kaum noch möglich wären.

${ }^{19}$ Die Bezeichnungen sind hier wie immer unklar und umstritten. Viele unterscheiden strikt zwischen Sozial- und Gesellschaftstheorie (Lindemann 2008; Münch 2004c). Joas und Knöbl (2004) beispielsweise
} 
des Theoretisierens ist es jedoch nicht zweckmäßig, prinzipielle Unterschiede zwischen Sozialund Gesellschaftstheorie auszumachen. In beiden Bereichen geht es um allgemeine Theoriebildung, der wesentliche Unterschied liegt in ihrem Gegenstand, wobei die jeweiligen Gegenstände sich wiederum jedoch nicht im Abstraktionsgrad oder in der Reichweite unterscheiden. Die Trennung von Sozial- und Gesellschaftstheorie wird auch von Theorien selbst unterlaufen, die sich zugleich als Sozial- und auch als Gesellschaftstheorie verstehen. Theorie der Gesellschaft und Theorie des Sozialen zugleich ist beispielsweise klassisch Max Weber mit seiner Handlungstheorie und der gesellschaftlichen Differenzierung in Wertsphären (Weber 1972, 1988), aber auch Luhmanns Systemtheorie als Kombination von allgemeiner Kommunikations- und Systemtheorie sowie der Differenzierungstheorie $(1984,1997)$ und Bourdieus Praxeologie $(1993,2013)$ changieren zwischen sozialtheoretischer Grundlegung und differenzierungstheoretischer Analyse. Insbesondere Bourdieus Habitus-Feld-Theorie zeigt dabei einerseits auf, dass soziale Felder die Habitūs der Akteure beeinflussen (Bourdieu 2013) und dass Felder den Akteuren Motivationen vorgeben (siehe für die entsprechende Weiterentwicklung der Feldtheorie Martin 2011). Andererseits fußt die feldtheoretische Differenzierungstheorie auf der allgemeinem Praxistheorie, da Felder wie Politik, Religion und Wissenschaft immer wieder durch entsprechende Praktiken reproduziert werden. Insofern sind hier - wie auch bei Weber und Luhmann - Gesellschafts- und Sozialtheorie aufs engste miteinander verknüpft, sodass sozialtheoretische Weiterentwicklungen immer auch Auswirkungen auf die Differenzierungstheorie haben und vice versa. ${ }^{20}$

Es lässt sich jedoch kein einheitliches Muster, keine dominante Praxis der sozial- und gesellschaftstheoretische Theoriebildung erkennen. Dennoch bilden häufig die „Klassiker“ der Soziologie und klassische Fragestellungen einen Ausgangspunkt, sodass Interpretationen und Analysen unterschiedlicher Art, Theoriekritik, hermeneutische Rekonstruktionen oder auch Theorieexegesen ihre besondere Berechtigung haben (Abend 2008: 179). In diesem Praxisfeld der Theoriebildung ist der Abstand zur konkreten empirischen Forschung am weitesten. Dies heißt jedoch nicht, dass die Arbeit an konkreten empirischen Problemen und Fragestellungen

betiteln ihre Einführung in soziologischen Theorien hingegen ganz bewusst mit Sozialtheorie und behandeln auch gesellschaftstheoretische Fragestellungen.

${ }^{20}$ Ferner sind Bourdieus feldtheoretische Arbeiten ein gutes Beispiel dafür, wie in diesem Modus sozialund differenzierungstheoretische Erkenntnisse mit empirischer Forschung verknüpft sind: Bourdieu gewinnt den Ansatz seiner Feldtheorie aus Webers Religionssoziologie (Bourdieu 2011a, 2011b) und nutz diese Erkenntnisse wieder in der empirischen Forschung, etwa in seinen Studien zum künstlerischen Feld (Bourdieu 2001). 
nicht bis zur Formulierung allgemeiner Sozial- und Gesellschaftstheorien führen kann. Goffman (2011) zeigt beispielsweise, wie man mittels „Beobachtungen“ (Alexander 1987) zur eigenen Sozialtheorie gelangen kann. Wohl lässt sich auch das Bemühen Bruno Latours (2014) um die Einführung der Akteur-Netzwerk-Theorie in den Kanon der Sozial- und Gesellschaftstheorie hier verorten, die zunächst als „spezielle Theorie“ im Bereich der Wissenschaftsforschung entstanden ist - und erst im Laufe der Zeit entwickelte sich daraus ein größerer Generalisierungsanspruch.

Modus 6 - Die Reflexion grundlegender Prinzipien und methodologischer Annahmen der Soziologie: Es lässt sich darüber streiten, ob es in der Soziologie allgemeinere Formen des Theoretisierens gibt als die Arbeit an Sozial- und Gesellschaftstheorien. Jedoch fließen allgemeine methodologische, erkenntnistheoretische und konzeptionelle Grundüberlegungen immer auch in die Formulierung von Sozial- und Gesellschaftstheorien ein. Man denke etwa an Kants Werttheorie bei Weber, an die phänomenologische Grundlegung der Wissenssoziologie oder an den Pragmatismus als Grundlagentheorie des Symbolischen Interaktionismus. In der Regel bilden allgemeine philosophische Theoreme und methodologische Grundüberlegungen oder die Auseinandersetzung mit vorherrschenden Paradigmen und Propositionen den Ausgangspunkt für Sozial- und Gesellschaftstheorien, aber auch für andere Modi des Theoretisierens. Ein prominentes Beispiel wäre hier die Auseinandersetzung von Bourdieu mit dem Strukturalismus, insbesondere seine Kritik der strukturalen Anthropologie von Levi-Strauss, sowie dem Existentialismus Sartres, aus dem Bourdieu schließlich seine "Theorie der Praxis" formuliert (Bourdieu 1993, 2009). Sozial- und gesellschaftstheoretische Konzeptionen bewegen sich damit sehr häufig im Schnittfeld zu allgemeinen Theoriediskussionen. Die Auseinandersetzung mit „Gerüsttheorien“ oder "Supertheorien“ (vgl. Haller 1999) ist jedoch nicht auf die Soziologie oder Philosophie beschränkt, denn auch Konzeptionen aus anderen Forschungsfeldern (etwa aus der Physik oder anderen Naturwissenschaften) können Grundlage des Theoretisierens sein. Man denke hier an Luhmanns Autopoiesis-Konzept (Luhmann 1984) oder an Beschleunigung und Resonanz bei Rosa $(2005,2019)$.

Die Reflexion soziologisch-theoretischer Grundlagen durch wissenschaftstheoretische Basisannahmen, epistemologische Erörterungen sowie grundlegende methologische Fragestellungen kommen in der Regel auch nicht ganz ohne Empiriebezug aus. Sie sind jedoch ein stückweit unabhängig von konkreten empirischen Forschungsfeldern; sie sind auch kaum 
mithilfe von empirischen Phänomenen falsifizierbar. Insofern bilden sie einen eigenen Bezugspunkt und ein eigenes Feld des Theoretisierens. Ein prominentes Beispiel für diese Art von Theoriearbeit wäre Karl Poppers Wissenschaftslehre und deren Anwendung sowie Übertragung in die soziologische Forschungspraxis. Andere Beispiele sind Abbotts Reflexionen zur Logik soziologischer Forschung und zur prozessorientierten Soziologie (Abbott 2007, 2016), zu denken wäre aber auch die Kontroverse um eine mikro- oder makrosoziologische Grundlegung der Soziologie (Alexander et al. 1987). Auch diese Schriften lassen sich als Vorarbeiten zur Formulierung von Sozial- und Gesellschaftheorien begreifen.

\section{Fazit}

Unser Anliegen war es, die Grundlagen soziologischer Theoriebildung zu erörtern und die verschiedenen Modi des Theoretisierens herauszuarbeiten. Im Gegensatz zu einer schematischen Vorstellung von empirischer Theoriebildung begreifen wir die Theoriearbeit und Theoriebildung in der Soziologie als ein vielschichtiges Unterfangen, das sich je nach Bezugsproblemen und Abstraktionsniveaus in seiner Praxis stark voneinander unterscheidet. Beim Theoretisieren kommt es folglich immer darauf an, welche Art von Theorie man zum Ziel hat und wie konkret der Bezug von Empirie und Theorie ausgestaltet ist, ob also die Erkenntnismittel eher aus empirischen Beobachtungen oder eher aus theoretisch gewonnenen Argumenten bestehen. Auf dieser Grundlage differenzieren wir sechs Modi des Theoretisierens, die von der Erklärung empirischer Phänomene bis hin zu der Arbeit an grundlegenden Annahmen und Prinzipien der Soziologie reichen. Wir hoffen damit einen Beitrag zur soziologischen Selbstaufklärung über die eigene (auch: unsere) Theoriearbeit zu leisten, da das Theoretisieren bisher zumeist implizit blieb und auch vom Streit um die, richtige' soziologische Theorie überdeckt wurde. Gleichwohl kann unser Beitrag nicht der Endpunkt einer Debatte sein, sondern ganz im Gegenteil soll er vielmehr als Anregung für die Beschäftigung mit soziologischer Theoriearbeit verstanden werden.

Zwei mögliche Anschlüsse wollen wir zum Abschluss kurz umreißen. Erstens wäre ein noch deutlicherer Schwerpunkt auf den konkreten Umgang mit und der Arbeit an Theorien, das konkrete doing des Theoretisierens, zu legen. So müsste deutlicher herausgearbeitet werden, wie das Theoretisieren in actu vonstattengeht. Wie gestaltet sich der tatsächliche Vollzug des Theoretisierens aus? Diese konkrete Praxis hängt, so unsere Hypothese, ganz entscheidend vom jeweiligen Modus des Theoretisierens, von den unterschiedlichen Theorietypen, wie sie Abend 
(2008) dargestellt hat, sowie auch von der jeweiligen Bezugnahme (und Vermittlung) auf die empirische und metaphysische Umwelt ab (vgl. Alexander 1982). Zielt man beispielsweise auf eine Bearbeitung von sozial- oder gesellschaftstheoretischen Problemstellungen (Modus 4 und 5), wäre ein möglicher erster Schritt hierzu die Kritik bestehender Ansätze, sodass sich an eine Form der Theoriekritik denken ließe, die sich von etwa von der Hypothesengenerierung unterscheidet. Es wäre dann zu fragen, wodurch sich diese Praxis - als Praxis! - auszeichnet, wie das konkrete doing einer Theoriekritik zu fassen ist: Was machen Theoretisierende genau, wenn sie Theorien kritisieren (oder auch an einer normativen Theorie arbeiten)? Um diesen Ansatz weiter zu verfolgen, wäre auch an ein ethnografisches Vorgehen zu denken, das gerade das Implizite des Theoretisierens explizieren und damit sichtbar machen kann. Ferner wäre, wenn man praxistheoretisch vorgeht, auch die Körperlichkeit des Theoretisierens mit zu adressieren (siehe zur Körperlichkeit sozialer Praxis Bourdieu 2013; Schmidt 2012), insofern ließe sich beim Theoretisieren sogar von einer Körpertechnik im Sinne Marcel Mauss' (1973) sprechen. Ein notwendiger nächster Schritt wären empirisch-konzeptionelle Studien zum doing im dargestellten ersten Modus, da uns diese konkrete Art und Weise des Theoretisierens noch immer als Rätsel gegenübertritt.

Zweitens, und aufbauend auf den eben skizzierten Studien, wäre die Leerstelle, dass Theoretisieren nicht gelehrt wird, zu füllen. Theoriearbeit als doing der Theoriebildung müsste Teil der (Theorie-)Lehre in der Soziologie werden, ganz ähnlich wie man die unterschiedlichen Methoden der empirischen Sozialforschung lehrt. Wir zeigen unseren Studierenden zwar sehr genau und eingehend, wie man von quantitativen Daten zu statistisch gehaltvollen Aussagen kommt oder wie man mithilfe der Objektiven Hermeneutik latente Sinnstrukturen aufdeckt. Es fehlt jedoch ein äquivalentes Vorgehen im Umgang mit Theorien beziehungsweise bei der Praxis des Theoretisierens. Wir finden Ansätze dazu bei Martins „activity of theory-work“ (Martin 2015: 1), indem er überzeugend darlegt, wie Parsons' Theorie zu kritisieren ist. Dem zur Seite zu stellen wären unserer Ansicht nach Lehrbücher, wie wir sie in der Philosophie finden. Denn die philosophischen Wissenschaften, die sich ja gerade durch einen hohen Bezug zu Alexanders metaphysischer Umwelt auszeichnen, haben einen Konsens über eigene „Werkzeuge des Philosophierens" (Pfister 2013; Rosenberg 2009), die von Fragen, was ein Argument überhaupt ist, über den Analogieschluss bis hin zur Analyse von logischen Aussagen in Sätzen reichen. Werkzeuge des Theoretisierens wären aus unserer Sicht auch für die Lehre und Praxis der 
Theoriepraxis wünschenswert - eine genauere, empirische Analyse unserer eigenen soziologischen Arbeit an und mit Theorien könnte hierfür wiederum die Grundlage bilden. 


\section{Abbildungen:}

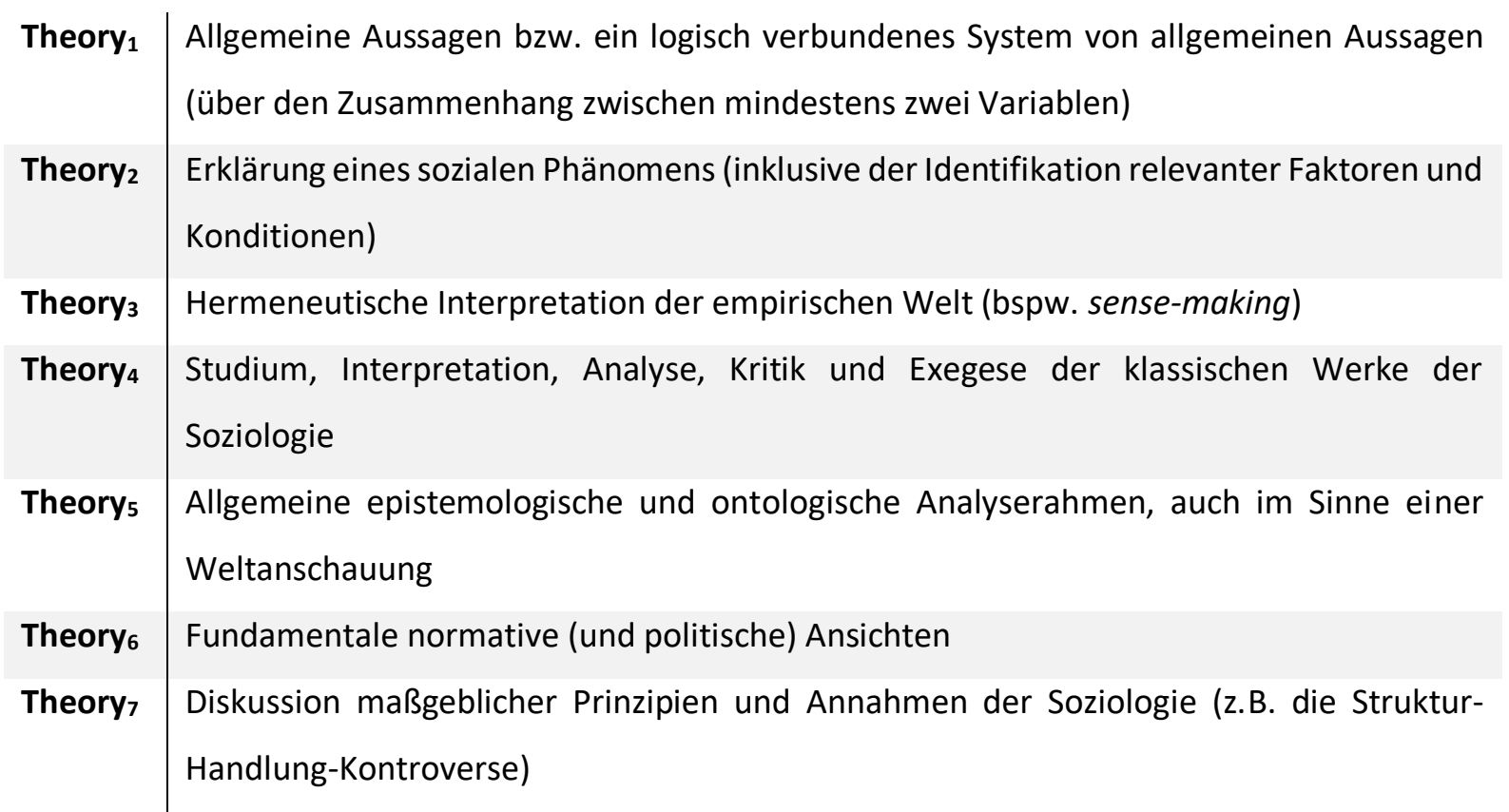

Abbildung 1: Die unterschiedlichen Bedeutungen von „Theorie“ nach Abend (2008). Eigene Darstellung aufbauend auf Abend 2008. Eigene Übersetzung.

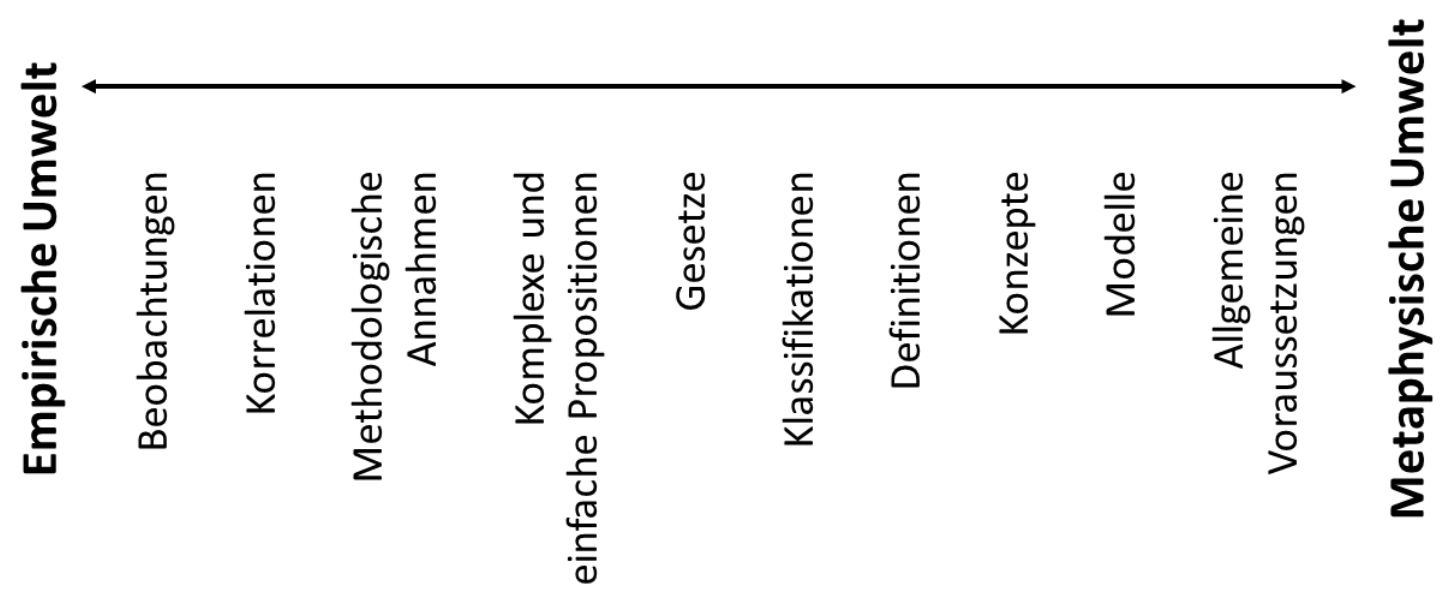

Abbildung 2: Das Kontinuum wissenschaftlicher Abstraktion nach Jeffrey Alexander. Eigene Darstellung und Übersetzung nach Alexander (1982: 3). 


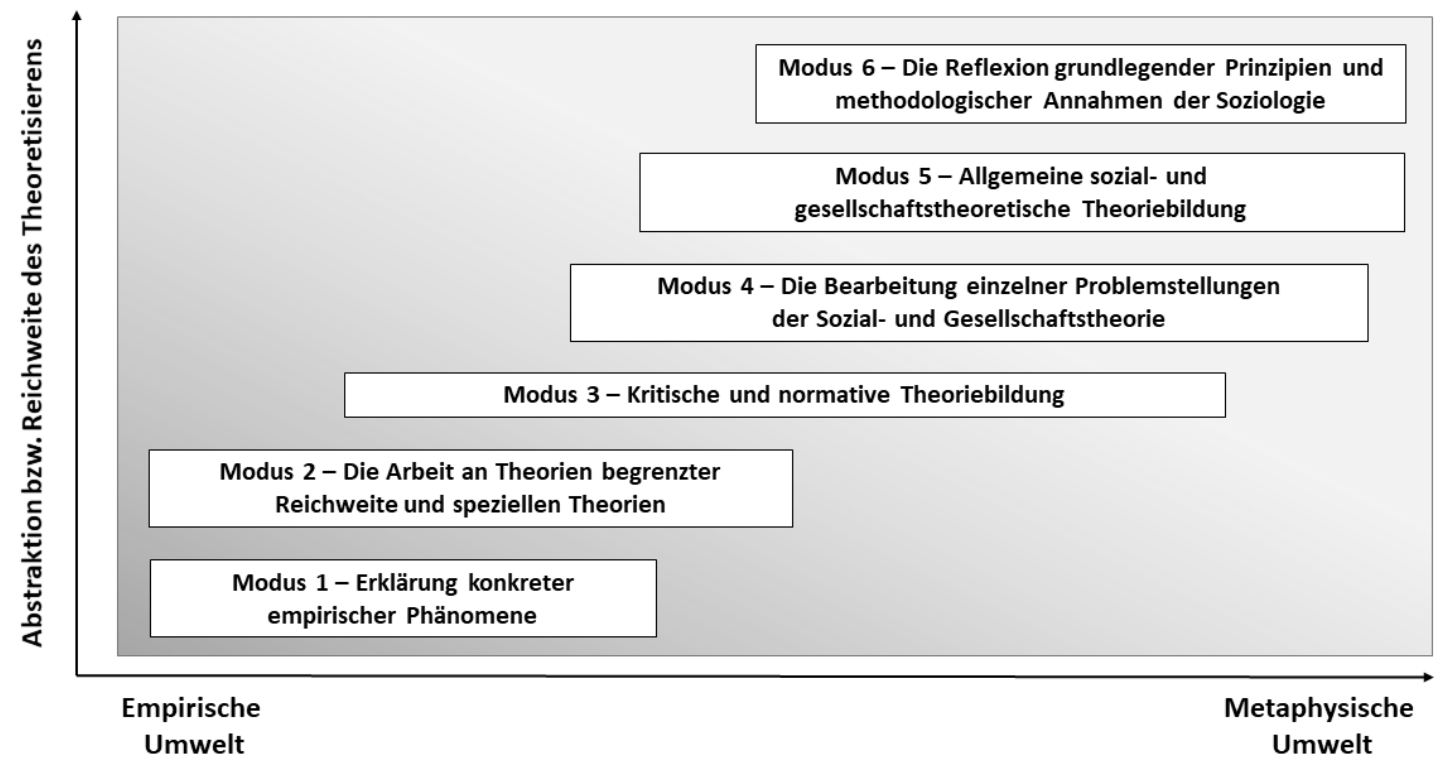

Abbildung 3: Modi des Theoretisierens. Eigene Darstellung. 


\section{Literatur}

Abbott, Andrew (2004): Methods of Discovery. Heuristics for the Social Sciences. New York/London: W. W. Norton.

Abbott, Andrew (2007): Chaos of disciplines. Chicago, Ill.: University of Chicago Press.

Abbott, Andrew (2016): Processual Sociology. Chicago/London: University of Chicago Press.

Abend, Gabriel (2008): »The Meaning of 'Theory' «. In: Sociological Theory 26(2), S. 173-199.

Abrahamson, Mark (1990): Sociological theory. An introduction to concepts, issues and research. Englewood Cliffs, NJ: Prentice Hall.

Adloff, Frank/Büttner, Sebastian M. (2013): „Die Vielfalt soziologischen Erklärens und die (UnJVermeidbarkeit des Eklektizismus«. In: Zeitschrift für Theoretische Soziologie (2), S. 253-267.

Albert, Gert/Sigmund, Steffen (Hg.) (2011): Soziologische Theorie kontrovers. Wiesbaden: VS Verlag für Sozialwissenschaften.

Alexander, Jeffrey C. (1982): Theoretical logic in sociology. Volunme One: Positivism, presuppositions, and current controversies. Berkeley: University of California Press.

Alexander, Jeffrey C. (1987): Twenty lectures. Sociological theory since World War II. New York: Columbia University Press.

Alexander, Jeffrey C./Giesen, Bernhard/Münch, Richard/Smelser, Neil J. (Hg.) (1987): The micro-macro link. Berkeley: University of California Press.

Anicker, Fabian (2019): "Theorie aus der Froschperspektive: zu Richard Swedbergs „theorizing" ". In: Zeitschrift für Theoretische Soziologie 8(2), S. 282-288.

Anicker, Fabian (2020): "Theoriekonstruktion durch Theorienvergleich - eine soziologische Theorietechnik«. In: KZfSS Kölner Zeitschrift für Soziologie und Sozialpsychologie, S. 1-30.

Atteslander, Peter (2010): Methoden der empirischen Sozialforschung. Berlin: Erich Schmidt Verlag.

Bachelard, Gaston (2016): Die Bildung des wissenschaftliches Geistes. Beitrag zu einer Psychoanalyse der objektiven Erkenntnis. Frankfurt am Main: Suhrkamp.

Benzecry, Claudio E./Krause, Monika/Reed, Isaac Ariail (Hg.) (2017): Social theory now. Chicago/London: University of Chicago Press.

Bourdieu, Pierre (1993): Sozialer Sinn. Kritik der theoretischen Vernunft. Frankfurt am Main: Suhrkamp. Bourdieu, Pierre (2001): Die Regeln der Kunst. Genese und Struktur des literarischen Feldes. Frankfurt am Main: Suhrkamp.

Bourdieu, Pierre (2009): Entwurf einer Theorie der Praxis auf der ethnologischen Grundlage der kabylischen Gesellschaft. Frankfurt am Main: Suhrkamp.

Bourdieu, Pierre (2011a): Eine Interpretation der Religion nach Max Weber. Berlin: Suhrkamp.

Bourdieu, Pierre (2011b): Genese und Struktur des religiösen Feldes. Berlin: Suhrkamp.

Bourdieu, Pierre (2013): Meditationen. Zur Kritik der scholastischen Vernunft. Frankfurt am Main: Suhrkamp.

Braun, Norman (2008): "Theorie in der Soziologie«. In: Soziale Welt 59(4), S. 373-395.

Bunge, Mario (1996): Finding philosophy in social science. New Haven/London: Yale University Press.

Coleman, James Samuel (1990): Foundations of Social Theory. Cambridge, Mass.: Belknap Press of Harvard Univ. Press.

Diekmann, Andreas (2018): Empirische Sozialforschung. Grundlagen, Methoden, Anwendungen. Reinbek bei Hamburg: Rowohlt.

Diekmann, Andreas/Voß, Thomas (Hg.) (2004): Rational-Choice-Theorie in den Sozialwissenschaften. Anwendungen und Probleme. München: Oldenbourg.

Dillon, Michele (Hg.) (2003): Handbook of the Sociology of Religion. Cambridge, UK/New York: Cambridge University Press.

Elster, Jon (2010): Explaining social behavior. More nuts and bolts for the social sciences. Cambridge: Cambridge University Press.

Esser, Hartmut (1993): Soziologie: Allgemeine Grundlagen. Frankfurt am Main [u.a.]: Campus.

Esser, Hartmut (2011): »Das Modell der Frame-Selektion. Eine allgemeine Handlungstheorie für die Sozialwissenschaften?». In: Albert, Gert/Sigmund, Steffen (Hg.): Soziologische Theorie kontrovers. Wiesbaden: VS Verlag für Sozialwissenschaften, S. 45-62. 
Farzin, Sina/Laux, Henning (2016): "Gründungsszenen - Eröffnungszüge des Theoretisierens am Beispiel von Heinrich Popitz' Machtsoziologie«. In: Zeitschrift für Soziologie 45(4), S. 241-260.

Giddens, Anthony (1988): Die Konstitution der Gesellschaft. Grundzüge einer Theorie der Strukturierung. Frankfurt/Main: Campus.

Giddens, Anthony/Turner, Jonathan H. (Hg.) (1988): Social theory today. Stanford: Stanford University Press.

Glaser, Barney G./Strauss, Anselm L. (1967): The discovery of grounded theory. Strategies for qualitative research. Chicago: Aldine.

Goffman, Erving (2011): Wir alle spielen Theater. Die Selbstdarstellung im Alltag. München: Piper.

Habermas, Jürgen (1995): Theorie des kommunikativen Handelns. 2 Bde. Frankfurt am Main: Suhrkamp.

Haller, Max (1999): Soziologische Theorie im systematisch-kritischen Vergleich. Opladen: Leske + Budrich.

Hedström, Peter (2008): Anatomie des Sozialen - Prinzipien der analytischen Soziologie. Wiesbaden: VS Verlag für Sozialwissenschaften.

Hedström, Peter/Ylikoski, Petri (2010): „Causal Mechanisms in the Social Sciences«. In: Annual Review of Sociology 36(1), S. 49-67.

Hempel, Carl G./Oppenheim, Paul (1948): „Studies in the Logic of Explanation«. In: Philosophy of Science 15(2), S. 135-175.

Honneth, Axel (1994): Kampf um Anerkennung. Zur moralischen Grammatik sozialer Konflikte. Frankfurt am Main: Suhrkamp.

Joas, Hans/Knöbl, Wolfgang (2004): Sozialtheorie. Zwanzig einführende Vorlesungen. Frankfurt am Main: Suhrkamp.

Kaesler, Dirk (Hg.) (2003): Klassiker der Soziologie. Von Auguste Comte bis Alfred Schütz. München: C.H. Beck.

Kaesler, Dirk (Hg.) (2005): Aktuelle Theorien der Soziologie. Von Shmuel N. Eisenstadt bis zur Postmoderne. München: Beck.

Kaesler, Dirk (Hg.) (2007): Klassiker der Soziologie. Von Talcott Parsons bis Anthony Giddens. München: C.H. Beck.

Kalthoff, Herbert/Hirschauer, Stefan/Lindemann, Gesa (Hg.) (2008): Theoretische Empirie. Zur Relevanz qualitativer Forschung. Frankfurt am Main: Suhrkamp.

Kant, Immanuel (1998): Kritik der reinen Vernunft. Hamburg: Meiner.

Karafillidis, Athanasios (2017): „Die Materie der Kybernetik. Über Kommunikation in organischmechanischen Verbindungen«. In: BEHEMOTH A Journal on Civilisation 10(1), S. 130-153.

Kneer, Georg/Schroer, Markus (Hg.) (2009): Handbuch soziologische Theorien. Wiesbaden: VS Verlag für Sozialwissenschaften.

Knoblauch, Hubert (2017): Die kommunikative Konstruktion der Wirklichkeit. Wiesbaden: Springer VS.

Krause, Monika (2016): »The meanings of theorizing". In: The British journal of sociology 67(1), S. 2329.

Kromrey, Helmut/Roose, Jochen/Strübing, Jörg (2016): Empirische Sozialforschung. Modelle und Methoden der standardisierten Datenerhebung und Datenauswertung mit Annotationen aus qualitativ-interpretativer Perspektive. Konstanz/München: UVK; UVK/Lucius.

Kroneberg, Clemens (2011): Die Erklärung sozialen Handelns. Grundlagen und Anwendung einer integrativen Theorie. Wiesbaden: VS Verlag für Sozialwissenschaften.

Kuhn, Thomas S. (1976): Die Struktur wissenschaftlicher Revolutionen. Frankfurt am Main: Suhrkamp.

Latour, Bruno (2014): Eine neue Soziologie für eine neue Gesellschaft. Einführung in die Akteur-

Netzwerk-Theorie. Frankfurt am Main: Suhrkamp.

Lindemann, Gesa (2008): "Theoriekonstruktion und empirische Forschung ". In: Kalthoff, Herbert/ Hirschauer, Stefan/Lindemann, Gesa (Hg.): Theoretische Empirie. Zur Relevanz qualitativer

Forschung. Frankfurt am Main: Suhrkamp, S. 107-128.

Luhmann, Niklas (1984): Soziale Systeme. Grundriß einer allgemeinen Theorie. Frankfurt am Main: Suhrkamp.

Luhmann, Niklas (1997): Die Gesellschaft der Gesellschaft. Frankfurt am Main: Suhrkamp. 
Martin, John Levi (2011): The Explanation of Social Action. Oxford: Oxford University Press.

Martin, John Levi (2015): Thinking through theory. New York u.a.: Norton.

Mauss, Marcel (1973): "Techniques of the body«. In: Economy and Society 2(1), S. 70-88.

Merton, Robert King (1995): Soziologische Theorie und soziale Struktur, hg. v. Meja, Volker/Stehr, Nico (Beister, Hella). Berlin [u.a.]: de Gruyter.

Morel, Julius/Bauer, Eva/Meleghy, Tamas/Niedenzu, Heinz-Jürgen/Preglau, Max/Staubmann, Helmut

(1989): Soziologische Theorie. Abriß der Ansätze ihrer Hauptvertreter. München: Oldenbourg.

Münch, Richard (2004a): Soziologische Theorie. Band 1: Grundlegung durch die Klassiker.

Frankfurt/M./New York: Campus.

Münch, Richard (2004b): Soziologische Theorie. Band 2: Handlungstheorie. Frankfurt/New York:

Campus.

Münch, Richard (2004c): Soziologische Theorie. Band 3: Gesellschaftstheorie. Frankfurt/Main: Campus.

Parsons, Talcott (1951): The social system. Glencoe, Illinois: Free Press.

Parsons, Talcott (1968): The structure of social action. New York: Free Press.

Pfister, Jonas (2013): Werkzeuge des Philosophierens. Stuttgart: Reclam.

Polanyi, Michael (1985): Implizites Wissen. Frankfurt am Main: Suhrkamp.

Popper, Karl R. (1984): Kübelmodell und Scheinwerfermodell: zwei Theorien der Erkenntnis. Hamburg:

Hoffmann und Campe.

Popper, Karl R. (2005): Logik der Forschung. Tübingen: Mohr Siebeck.

Reckwitz, Andreas (2006): Die Transformation der Kulturtheorien. Zur Entwicklung eines

Theorieprogramms. Weilerswist: Velbrück.

Reichenbach, Hans (1961): The Rise of Scientific Philosophy. Berkeley: University of California Press.

Rosa, Hartmut (2005): Beschleunigung. Die Veränderung der Zeitstrukturen in der Moderne. Frankfurt am Main: Suhrkamp.

Rosa, Hartmut (2019): Resonanz. Eine Soziologie der Weltbeziehung. Berlin: Suhrkamp.

Rosa, Hartmut/Strecker, David/Kottmann, Andrea (2009): Soziologische Theorien. Konstanz: UVK.

Rosenberg, Jay F. (2009): Philosophieren. Ein Handbuch für Anfänger. Frankfurt am Main: Vittorio Klostermann.

Scheer, Monique (2012): „Are emotions a kind of practice (and is that what makes them have a history)? A Bourdieuian approach to understanding emotion«. In: History and Theory 51(2), S. 193220.

Schmidt, Robert (2012): Soziologie der Praktiken. Konzeptionelle Studien und empirische Analysen. Berlin: Suhrkamp.

Schnell, Rainer/Hill, Paul B./Esser, Elke (2011): Methoden der empirischen Sozialforschung. München: Oldenbourg.

Schroer, Markus (2017): Soziologische Theorien. Von den Klassikern bis zur Gegenwart. Paderborn: Wilhelm Fink.

Silver, Daniel (2019): "Theorizing Is a Practice, You Can Teach It«. In: Canadian review of sociology = Revue canadienne de sociologie 56(1), S. 130-133.

Skirbekk, Gunnar (Hg.) (1977): Wahrheitstheorien. Eine Auswahl aus den Diskussionen über Wahrheit im 20. Jahrhundert. Frankfurt am Main: Suhrkamp.

Strauss, Anselm L. (1998): Grundlagen qualitativer Sozialforschung. Datenanalyse und Theoriebildung in der empirischen soziologischen Forschung. München: Wilhelm Fink.

Swedberg, Richard (2012a): "On Charles S. Peirce's Lecture "How to Theorize" (1903)«. In: Sociologica (2), S. 1-27.

Swedberg, Richard (2012b): "Theorizing in sociology and social science. Turning to the context of discovery". In: Theory and Society 41(1), S. 1-40.

Swedberg, Richard (2014a): The Art of Social Theory. Princeton, New Jersey: Princeton University Press.

Swedberg, Richard (Hg.) (2014b): Theorizing in social science. The context of discovery. Stanford, Calif.: Stanford Social Sciences.

Swedberg, Richard (2016): „Before theory comes theorizing or how to make social science more interesting". In: The British journal of sociology 67(1), S. 5-22. 
Swedberg, Richard (2017): "Social Theory and Theorizing". In: Turner, Bryan S. (Hg.): The Wiley-

Blackwell Encyclopedia of Social Theory. Oxford, UK: John Wiley \& Sons, S. 1-13.

Treibel, Annette (1997): Einführung in soziologische Theorien der Gegenwart. Opladen: Leske + Budrich.

Turner, Jonathan H. (1986): The structure of sociological theory. Belmont: Wadsworth Publ.

Turner, Jonathan H. (Hg.) (2001): Handbook of sociological theory. New York: Springer.

Turner, Jonathan H. (2012): Contemporary sociological theory. Los Angeles: Sage.

Villa, Paula-Irene (2009): „Feministische- und Geschlechtertheorien«. In: Kneer, Georg/Schroer, Markus

(Hg.): Handbuch soziologische Theorien. Wiesbaden: VS Verlag für Sozialwissenschaften, S. 111132.

Weber, Max (1972): Wirtschaft und Gesellschaft. Grundriss der verstehenden Soziologie. Tübingen: J. C.

B Mohr (Paul Siebeck).

Weber, Max (1988): Gesammelte Aufsätze zur Religionssoziologie I. Tübingen: Mohr.

Weiß, Johannes (2017): »Jungbrunnen der Theorie? Über Entdeckungskontexte und

Gründungsszenen«. In: Soziologische Revue 40(2), S. 244-253. 